\title{
ON THE FORMALIZATION OF PROGRAMMING LANGUAGES: \\ EARLY HISTORY AND MAIN APPROACHES
}

\author{
Peter Lucas
}

\section{Absstraact:}

The paper discusses the nature of the subject, and summarizes its origins. The main approaches and their interrelationships are discussed. The author's view on the long and short range objectives is presented. 


\section{CONTENTS}

1. On the Significance of the Area

2. Historical Background 6

3. Basic Methodological Approaches 10

3.1 Abstract syntax 10

3.2 Mathematical Semantics 11

3.3 Operational Semantics 15

$\begin{array}{lll}3.4 & \text { Axiomatic Approach } & 18\end{array}$

4. Challenges 2I-23 


\section{ON THE SIGNIFICANCE OF THE AREA}

Computer systems can be viewed as machines capable of interpreting languages; they accept and understand declarative sentences, obey imperative sentences and answer questions, all within the framework of those languages for which the systems were built. A computer system accomplishes its tasks on the basis of a prescription of these tasks, i.e. on the basis of a program expressed in some programing language.

There is no inherent disparity between human languages (including natural language and the artificial languages of science) and languages used to talk to computers. Thus there is no need to apologize for "anthropomorphisms" in the above point of view; in fact our only way to talk scientifically about the relation of humans to their natural languages is in terms of computer notions (or so it seems to me).

By viewing computers as language interpreting machines it becomes quite apparent that the analysis of programing (and human) languages is bound to be a central theme of Computer science.

Part of the fascination of the subject is of course related to its intimate connection to human language, i.e. the mechanisms we study mirror in some way at least part of our own internal mechanisms.

Although there is no inherent disparity between human language and computer language, there is at present a huge gap between what we can achieve by human conversation and our communication with machines. A little further analysis will indicate the nature of the gap.

First we consider the structural aspect of language, i.e. how phrases are composed of words and sentences are built from phrases, commonly called "syntax". There are efficient and precise methods to define the syntax of a language and algorithms to compose and decompose sentences according to such definitions. The problem is more or less solved. Yes, computer language usually have a simpler and more regular syntax than natural languages (as even some scientific notations) and there are technical problems yet to be solved. Yet, it seems to me, there is not much of a gap.

Second, there is the aspect of meaning, or "semantics" as it is usually called. Now we get into more subtle problems. Let me restriat the discussion, for the time being, to the objects we can talk about in the 
various languages (rather than considering what we can say about them). Programming languages in the strict sense talk invariably about rather abstract objects such as numbers, truth-values, character strings and the like. Certainly, the major programing languages in use do not let us talk about tables, chairs or people, not even about physical dimensions of numbers such as: hours, pounds or feet. The commercial languages do not know about the distinction of dollars and francs, and scientific languages do not know about time and space. There have been some attempts to include those notions or a device that makes it possible to define these notions within a language, e.g. the class concept in SIMULA and PASCAL and the investigations around abstract data types. If we extend the notion of programing language to include query languages and database languages we may observe a tendency in the indicated direction. Yet, there is a gap. Artificial Intelligence has experimented for some time with languages that can be used to talk about objects other than numbers etc.; we should probably look a lot more frequently accross the fence.

Definition methods concerning semantic, and even more so, mechanical ways to use semantic definitions are much less understood than in the case of the syntactic aspect.

Thirdly, there is the aspect of language understanding; I hesitate to call this "pragmatics" since the latter term has been used for too many things.

Suppose I ride on a train with a friend. The friend observes: "The windows are wet" ${ }^{1}$ ). The statement is presumably structured according to the english grammar and has a certain meaning. However, I would probably not just analyze the sentence and determine its meaning, and leave it at that. Most likely I would react by looking at a window, observe that there are drops, conclude that it is raining, prepare my umbrella so that I don't get wet and ruin my coat when I get of the train, etc... To araw all these conclusions and act accordingly I need

(1) It would not make any difference to the following argument if my learned friend had used META-IV and passed a note saying: "wet (windows)". That is to say, I do not discuss the distinction between natural language and standard (formal) notation but the distinction of the human and computer use of the statement irrespective of the form. 
to use a lot of knowledge about the physical world in general and about my specific environment. It is in this area of language understanding, where I see the bigger gap between our interaction with the computer as opposed to humans. What is lacking in the machine are models of the external world and general mechanisms to draw conclusions and trigger actions. Again Artificial Intelligence and natural language research have been concerned with the problem. Yet, this has not had any practical influence on e.g. commercial applications. With the increase in computer power it might very well be worth looking over the fence.

With the preceding paragraphs I wanted to put the present subject into a much larger context than is usual. Thank God, there is more to programming languages than procedures, assignment and goto's (or no gotos).

The rest of the paper is a lot less ambitious and remains more or less within the traditional concepts of programring languages. It presents my subjective perception of the various origins of the methods of semantic definitions. Each of the three main approaches are then summarized. The paper concludes by outlining some more short range objectives. 


\section{HISTORICAL BACKGROUND}

The theory of programing languages, the related formal definition techniques in particular, has roots in - and is related to - several other disciplines such as linguistics, formal logic and certain mathematical disciplines. In fact, the terms "syntax" and "semantics" and with these terms the distinction between the respective aspects of language, have been introduced by the american philosopher charles Morris [Morris 38, Zemanek 66 ]. He developed a science of signs which he called semiotics. Semiotics, according to Morris, is subdivided into three distinct fields: syntax, semantics, and pragmatics. In his book on Signs, Language, and Behavior [Morris 55] Morris defines:

"pragmatics - deals with the origin, uses and effects of signs within the behavior in which they occur;

semantics - deals with the signification of signs in all modes of signifying;

syntax - deals with the combination of signs without regard for their specific significations or their relation to the behaviour in which they occur."

The clear distinciton between syntax and semantics, first applied to a programming language in the ALGOL 60 report [Naur 63], has turned out to be tremendously useful. There have been several not so successful attempts to carry the notion of pragmatics into the theory of programming languages (see e.g. San Dimas Conference [ACM 65]). We may start the history of formal definition methods for programming languages with the year 1959 when J. Backus proposed a scheme for the syntactic definition of ALGOL 60 [Backus 59]. This scheme (a generative grammar) was then actually used in the ALGOL 60 report; the related notation is known as BNF (for Backus Normal Form or Backus Naur Form). BNF, or variations thereof, have been used in many instances; it has stimulated theoretical research as well as practical schemes for compiler production (both automatic and non-automatic). Roughly speaking, BNF grammars coincide with the class of context free grammars of Chomsky [Chomsky 59]; it is worth mentioning that Chomsky divides his grammatical formalisms in an attempt to obtain a basis for the syntax of the English language. Much research has been devoted to the study of subtypes and extended types of BNF grammars; the latter in support of the desire to capture more syntactic properties of the language to be defined; the former, i.e. the study of subtypes is usually motivated 
by the wish to guarantee properties which permit fast syntax recognition and analysis algorithms. The subject of formal syntax definition, and the related computational problems and methods, have found their way into textbooks and computer science curricula; in fact, the larger part of compiler writing courses is usually spent on syntax problems.

At least since the ALGOL 60 report, the lack of rigorous definition methods for the semantics of programing languages was widely recognized; furthermore, the success of formal syntax definitions invited similar attempts for the semantic aspects of programing languages, yet, the problem turned out to be of an obstinate nature. To date, there is no satisfactory solution, at least none that enjoys the consensus of the computing community.

The instructions of machine languages are defined by the behaviour of the respective machine upon execution of these instructions. The associated manuals usually describe first what constitutes the state of the specific machine (e.g. content of main storage, content of registers, etc.) and then for each instruction and any given state the successor state after execution of the instruction to be defined. Hence, for a programmer, the most direct way to define a programming language is in terms of an interpreting machine; only that, for higher level languages, we must abstract from particularities of hardware machines and inplementation details and use a suitable hypothetical machine instead. E.W. Dijkstra formulated the situation in 1962 [Dijkstra 62] as follows: "A machine defines (by its very structure) a language, viz. its input language; conversely, the semantic definition of a langauge specifies a machine that understands it"l).

The classic paper that has triggered a large volume of follow-on work is by McCarthy [MeCarthy 63]. The paper outlines a basis for a theory of computation; more important for our subject, it establishes the main goals and motivation: methods to achieve correctness of programs in general and of compilers in particular; rigorous language definitions constitute a subgoal. The schema for language definitions proposed by McCarthy contains a number of novel subjects. Firstly, a complete se-

(1) it would be unfair to include this quotation and not say that prof. E.W. Dijkstra would probably no longer defend this position, and rather tend to be a proponent of the direction described under "Axiomatic Approach" in this paper. 
paration of notational issues, i.e. the representation of phrases by linear character strings, from the definition of the essential syntactic structure of a language. The latter definition is called Abstract syntax. It is, at least for complicated languages, much more concise than the concrete syntax. Thus, a semantic definition on the basis of an abstract syntax becomes independent of notational details and is also more concise. Secondly, state vectors are introduced as the bases of the semantic definitions proper, i.e. the meaning of an instruction or statement is defined as a state transition. The paper shows in principle the task of proving compilers correct. The basic scheme of language definitions has been elaborated in many instances during the past decade, e.g. by the earlier work of the Vienna Laboratory on PL/I [Lucas 69] and the proposed ECMA-ANSI standard [ECMA 74].

Another successful direction of research was initiated by $P$. Landin [Landin 64,65], using the lambda-calculus [Church 41] as the fundamenta1 basis. He revealed that certain concepts of ALGOL 60 (and similar languages) can be viewed as syntactic variations (syntactic sugar) of the lambda-calculus. The inherently imperative concepts, assignment and transfer of control, were captured by introducing new primitives into the lambda-calculus; the extended base is defined by the so-called SECD machine, a hypothetical machine whose state consists of 4 components: Storage, Environment, Control and Dump. The machine state has more structure than the state vectors of McCarthy, because the machine had to reflect more complicated concepts (blocks, local names) than McCarthy's original simple example was intended to.

In 1964 [Strachey 66] c. Strachey argued that, with the introduction of a few basic concepts, it was possible to describe even the imperative parts of a programming language in terms of the lambda-calculus. With the referenced paper, $c$. Strachey initiated a development that led to an explication of programming languages known as mathematical or denotational semantics. The fundamental mathematical basis for the latter development was contributed by $D$. Scott in 1970 [Scott 70]. The joint paper, by $D$. Scott and $C$. Strachey [Scott 71] offers a description method and its application to essential language concepts based upon the indicated research.

Research on axiom systems and proof theory suitable as a base for correctness proofs of programs was initiated by $R$. Floyd [Floyd 67], with a simple flow diagram language. C.A.R. Hoare [Hoare 69,71], extended and refined the results to apply to constructs of higher level languages. 
The area has been the most actively pursued, including expreriments in automatic program verification.

There are several pioneering research efforts, which do not so evidentIy fall into the categories introduced above. Among the very early results on semantics published is A. van Wijngaarden's "Generalized ALGOL" [ van Wijngaarden 62]. De Bakker [de Bakker 69] discovered that the schema proposed by $A$. van Wijngaarden can be viewed as a generalized Markov Algorithm. A. Carraciolo Forino 66] also used Markov Algorithms as the starting point for the formalization of programming language semantics.

For anyone familiar with syntax directed compilers it is tempting to apply similar ideas to the definition of semantics. A definition method on this basis is due to D. Knuth: Semantics of Context Free Languages [Knuth 68]. In some way or another, a formal definition of the semantics of a language invariably specifies a relation between any phrase of the language and some mathematical object called the denotation of the phrase. D. Knuth provides a convenient scheme that allows the specification of functions over the phrases of a language assuming that the phrase structure of the language is given by a production system.

Most research so far has been devoted to the definition and analysis of existing languages (or concepts found in existing languages). Yet, formal semantics could be a most valuable intellectual tool for the design of novel programming concepts (or synonymously: novel programming language constructs). There are rare instances of such applications of formal semantics (e.g. [Dennis 75, Henderson 75]). 


\section{BASIC METHODOLOGICAL APPROACHES}

\subsection{Abstract Syntax}

The notion of abtract syntax is of considerable value for practical definitions of notationally complicated languages. There exist several methodological variations, which all achieve the same objective: to abstract from semantically irrelevant notational detail and reduce the syntax to define the essence of the linguistic forms only.

For illustration consider the following examples. Let $v$ be the category of variables and $e$ be the category of expressions. Several notational variants are in use to denote assignment statements e.g.:

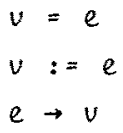

The semanticaliy essential structure common to these notations is: That there is a syntactic category called assignment statement, and that an assignment statement has two components, a variable and an expression.

An abstract syntax may define an expression to be either an elementary expression (variable, constant, etc.,) or a binary operation consisting of an operator, a first operand, and a second operand (the definition of expressions may have several other alternatives); operands are again expressions.

As a concrete syntax, meant to define character strings, such a definition would be hopelessly insufficient and ambiguous, $e . g$. we would not know whether to parse $x+y^{*} z$ into:
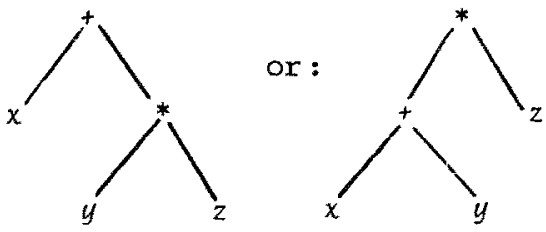
Thus the concrete syntax has to introduce punctuation marks such as parentheses, and, in the example of expressions, precedence rules of operators to avoid ambiguities. However, the definition of expressions given above is perfectly usable as an abstract syntax definition.

It can be regarded as a definition of parsing trees, hence the ambiguity problem is completely avoided. Thus there are advantages gained even in the case where only one language is considered: representational details are suppressed and each phrase is given a kind of normal form.

For practical cases, such as PL/I, the number of rules necessary to define an abstract syntax is much smaller than for the corresponding concrete syntax; hence we have obtained a more concise basis for the semantic definition. The price we pay is an additional part for the formalization of a language, which establishes the relation between the concrete and the abstract syntax.

\subsection{Mathematical Semantics}

The semantics of a given language is formalized by associating a suitable mathematical object (set, function, etc.) with each phrase of the language; the phrase is said to denote the associated object; the object is called the denotation of the phrase. Furthermore, to gain a view which is referentially transparent, of the language to be defined, denotations of composite phrases shall be defined solely in terms of the denotations of the subphrases. The major problem in establishing the mathematical semantics for a given language is to find suitable mathematical objects, that can serve as the denotations.

Using the notation introduced by $D$. Scott and $C$. Strachey we will write $I[p]$ for the denotation of a phrase $p^{1)}$. To indicate the various phrases to be discussed, we will use an ALGOL like and otherwise obvious notation, e.g. $I[x:=x-1]$ is the denotation of the assignment statement $x:=x-1$.

(1) for small examples, like those introduced in this paper, it is better to abstain from using abstract syntax and META-IV functions. For larger examples (e.g. PL/I), the notational conventions used here would lead to considerable difficulties. 
The further elaboration of the subject will go through a series of programming language concepts in increasing order of complexity. Take first a simple language with a fixed set of variables ( $i a j)$, expressions (e) without side effects, assignments statements $(i d:=e)$ and compound statements $(s 1 ; s 2)$. If we were to construct a definitional interpreter, we would certainly introduce a state vector (á lá McCarthy). Although we do not wish to specify particular ways to compute the effect of executing programs and their parts, we still have to characterize the overall effect of this execution. Therefore we introduce state vectors $\sigma$, which are (usually) partial functions from variable names ID into the set of values, VAL, which a variable may assume, i.e.

$$
\sigma: I D \vec{m} V A L
$$

Let $\Sigma$ be the set of all possible states. The kinds of denotations that occur in the example language can now be chosen as follows:

$$
\begin{aligned}
& I[e]: \Sigma \stackrel{\sim}{\rightarrow} V A L \\
& I[s t]: \Sigma \stackrel{\sim}{\rightarrow} \Sigma
\end{aligned}
$$

i.e. the denotations of expressions are functions from states into values and the denotationsof statements are functions from states to states.

Assuming that $I[e]$ has been defined elsewhere, the definition of assignment and compound statement according to the philosophy of mathematical semantics read:

$$
\begin{aligned}
& I[i d:=e](\sigma)=\begin{array}{l}
\text { assign }(\sigma, i d, I[e](\sigma)) \\
\text { where: } \operatorname{assign}(\sigma, i d, v a l)=\sigma^{\prime},
\end{array}, \sigma^{\prime}(x)=\left\{\begin{array}{l}
v a l \text { for } x=i d \\
\sigma(x) \text { for } x \neq i d
\end{array}\right. \\
& I[s 1 ; s 2]=I[s 2] \cdot I[s I] \quad \ldots \text { functional composition }
\end{aligned}
$$

Note that denotations of composite phrases are given in terms of denotations of immediate subphrases and that we have avoided introducing a statement counter. For each additional language feature we may have to revise the definition of states, introduce new ways to compose denotations or even design new kinds of mathematical objects.

As a first complication we introduce a loop statement of the form: while $e$ do s, say. We assume that e returns a truth value and intui- 
tively expect that the denotation of the loop statement can be defined as:

$$
I[\text { while e do } s](\sigma))= \begin{cases}I[\text { whize e do } s](I[s](\sigma)) & \text { if } I[e](\sigma) \\ & \text { if } I[e](\sigma)\end{cases}
$$

Evidently, the definition is of the form $f=F(f)$, with $f=I$ [white e do s], i.e. $f$ is defined as the fixed point of $F$. Before this definition can be accepted as meaningful, one has to ask whether such a fixed point always exists and whether it is unique. The existence can be asserted under appropriate mathematical restrictions (introducing concepts of monotonicity and continuity); there will in general be more than one fixed point satisfying the equation. Thus an additional rule has to be introduced which makes the defined object unique (the "smallest" fixed point under a suitably defined ordering relation). This is not the place to elaborate the issue at length and the reader must be referred to the respective literature.

However, it should by now be evident that we are led into deep mathematical issues, and this at a stage where, from a programing language point of view, we have only introduced the most primitive language constructs. At this point, it seems that we have to contemplate the potential uses of a semantic definition. The author suggests to distinguish between the foundation of the subject matter and more practical problems like the description of real-life programing languages for compiler writers. Like the foundations of mathematics on the one hand and applied mathematics on the other, these two fields are not unrelated but are distinct. If we accept the program of mathematical semantics, the steps we have tried to indicate follow, and the difficulties observed above are inevitable. On the other hand, it seems unrealistic and in fact unnecessary to require that each compiler writer be fluent in modern algebra. Rather, one would expect that the foundations are used to justify, once and for all, useful practical methods which in turn can be applied directly by the practitioner (not everybody who applies Fourier Analysis need to be a specialist in the foundations of mathematics).

Next we introduce expressions with side effect, i.e. evaluation of an expression returns a value and changes the state. Consequently, we change the type of object an expression denotes to:

$$
I[e]: \quad \Sigma \stackrel{\sim}{\rightarrow} \quad V A L
$$


The definition of assignment and loops are to be changed accordingly. A further important concept in most programming languages is that of local names, i.e. names which are declared for a specific textual scope of a program. The syntactic category is called block and takes the form: begin dcl id; st end.

Since the same name may now be used in different blocks for different purposes we have to introduce some device in the definition which enables us to distinguish the different uses of a name. One usually introduces an auxiliary object called environment, env, which is a function from names (variable names in the present example) to so called locations; the state then maps location into values. Thus a state $\sigma$ is now a function of type $\sigma: L O C \vec{m}_{\mathrm{m}} V A L$, where $L O C$ is a set of primitive objects called locations; the auxiliary object env is of type env:ID $\vec{m}_{\mathrm{m}} \mathrm{CC}$.

In order to interpret a given phrase we always have to have an environment which associates the names occurring in the phrase with locations. The mathematical types of the denotations have to be revised:

$$
I[e]: E N V \stackrel{\sim}{\rightarrow}(\Sigma \stackrel{\sim}{\rightarrow} \Sigma V A L)
$$

i.e. I $[e]$ when applied to an environment yields a function which transforms a state and returns a value.

$$
I[\underline{\text { begin }} \underline{\text { del }} \text { id;s end] }: E N V \stackrel{\sim}{\rightarrow}(\Sigma \sim \Sigma)
$$

The types of the denotations of the other constructs are designed similarly. The denotation of blocks, the new construct, is defined as:

$$
I\left[\underline{b e g i n} \text { del id;s end] }(e n v)(\sigma)=I[s]\left(e n v^{\prime}\right)\left(\sigma^{\prime}\right) \mid \text { restriet to dom of } \sigma\right.
$$
where $\sigma^{\prime}(x)=\left\{\begin{array}{lll}\Omega & \text { if } x=20 c \\ \sigma(x) & \text { otherwise }\end{array}\right.$ env $(x)=\left\{\begin{array}{lll}\text { Loc } & \text { if } & x=i d \\ \text { env }(x) & \text { otherwise }\end{array}\right.$

where: $20 c$ is some locELOC not in dom of $\sigma$

The lastfeatures to be discussed in this section are procedure declarations and parameter passing. What should the denotation of a procedure (in the sense of ALGOL or PL/I) be? According to the philosophy of mathematical semantics it is of type:

$$
E N V \rightarrow\left(\left(A 1 \ldots A h_{2}\right) \cong(\Sigma \simeq \Sigma)\right)
$$


i.e. given arguments $a 1, \alpha 2, \ldots, a n$, and env to interpret the global iden tifiers occurring in the procedure, the procedure is simply a state to state transition function. Some higher level programing languages permit procedures to be passed as arguments in particular a procedure can be passed as an argument to itself. In contrast, the type of a function is usually considered distinct from its range of arguments and value range to avoid the well-known paradoxes. The establishment of a type free theory of functions which provides the appropriate domain of denotations for procedures is a major achievement of $D$. Scott [Scott 70]. Apart from those language constructs which none of the methods has so far addressed, there are several problems which have as yet no known solution in the framework of mathematical semantics; in particular parallel and quasi-parallel execution (tasking, unspecified order of execution). Condition handling in $\mathrm{PL} / \mathrm{I}$; labels and goto's have been formulated, however, the models do not closely correspond to the intuitive concept of the construct. With parallel processing, it seems referential transparency cannot be achieved as long as denotations of statements merely reflect the initial state/result state relation. There are two sizable language definitions in this style. Firstly, a definition of ALGOL 60 [Mosses 74]. Secondly a recent definition of a subset of PL/I [Bekié 74].

There is an exellent tutorial on the subject by J. Stoy [Stoy 74 ].

\subsection{Operational Semantics}

The semantics of a programming language can be defined via a hypothetical machine which interprets the programs of that language; such methods have been called "operational", or "constructive". The latter term is, however, misleading, because the specification of hypothetical machines may contain non-constructive elements, such as quantifiers, implicit definitions and infinite objects. The term "definitional interpreter" is sometimes used instead of "hypothetical machine".

By machine we understand a structure consisting of a set of states, two subsets thereof: the initial states and the end states, a state transition function and a function which maps programs and their input data into initial states; also usually not given explicitly, there should be a function which takes end states as arguments and yields that part of 


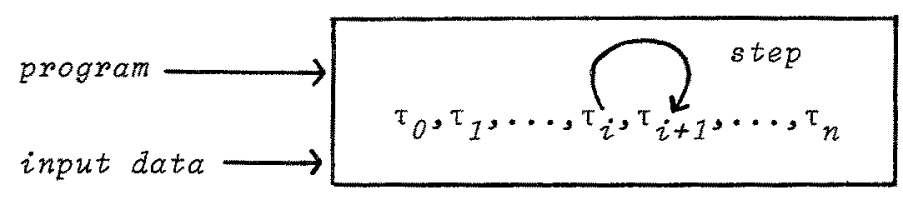

$\tau_{0} \quad \ldots$ initial state

step ... state transition function

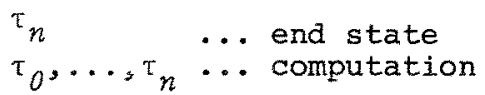

an end state which is the result of the program. Since most higher level languages are such that the program remains constant, i.e. is not modified, during its interpretation, one could also keep the program separate and only include a statement counter to the currently executed statement within the state itself. The definition of the step function, if properly done, will reflect the syntactic structure of the language, such that we cannot only relate an entire program to a computation, but also subphrases of the program to sections of the computation, i.e. we may ask what a specific subphrase in a given context means. In languages, like e.g. PL/I, where the order of operations is not entirely fixed, the defining hypothetical machine is non-deterministic, i.e. the step function will, in general, yield a set of possible successor states and a program will thus be related to a set of computations.

To illustrate the differences (and similarities) between mathematical semantics and operational methods consider the following example:

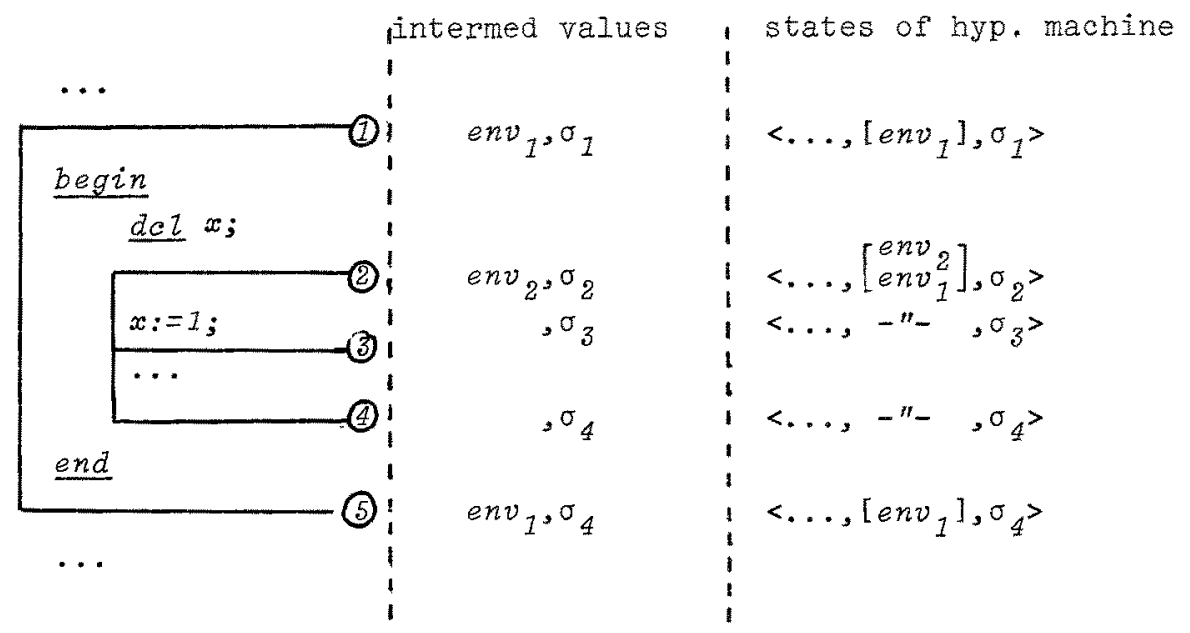


The example program part is a block with one declaration and a compound statement as the executable part. In order to elaborate this program part, given a mathematical definition, we would have to compute the intermediate states and environments shown in column one of the above table. The sequence of intermediate values almost represents the computaion of a hypothetical machine. The previously introduced concept of a machine requires that each state of a computation only depends upon its immediate predecessor state. Consequently, in order to have the environment available at point (5) of the computation it has to be remembered, i.e. contained in the states (2), (3) and (4). This is accomplished by an environment stack as shown in the second column of the above table; the stack is part of the state of the hypothetical machine.

The example shows that the state has to be extended in order to get from the mathematical semantics to a corresponding hypothetical machine; we have to construct a "grand state" as it is sometimes called. At the same time the discussed step indicates how one could get from a language definition, in a systematic fashion to the related implementation. More precisely, what remains to be done is to find a finite representation of the grand states in terms of data and storage structure of the target machine and the machine code which simulates the given transformation on the grand state.

In the relevant literature there exist various examples relating language definition to implenentaions (e.g. [McCarthy 67]). A comprehensive elaboration of this subject would be of great tutorial and practical value and would in fact complement the existing material on the subject of syntax definition and parsing, which, in contrast, is well understood. (Books on compiler writing have usually a lot to say on the syntactic part of the question and very little on semantics, object time organization and code generation.) The step from the syntax definition to the respective parser can be automatic. We are far from that stage on the semantic side of the problem.

The method has been applied to several large languages, in fact the proposed ECMA/ANSI PL/I standard [ECMA 74] has been formulated using an operational definition. The method is the only one presently known, which is capable of covering the currently existing language constructs.

There is a tutorial on the subject by A. Ollongren [01longren 75], and several summaries, e.g. an in depth evaluation by J.C. Reynolds [Reynolds 72 ] 


\subsection{Axiomatic Approach}

The essential semantics of a programing language is defined by a collection of axioms and rules of inference, which permits the proof of properties of programs, in particular that a given program is correct, i.e. realizes a specified input/output relation. Of course, one can prove assertions about programs using either a mathematical or operational definition and ordinary mathematical reasoning. In fact, the axioms and rules of inference can be regarded as theorems within the framework of mathematical semantics.

However, the objective of the axiomatic method is a fomal system which permits to establish proofs using the uninterpreted program text only, i.e. without referring to denotations of the program or program parts. Whenever we talk about denotations in this section, then this is for explanatory purposes and is not part of the axiomatic system.

The problem of correctness proofs of programs is usually split into two subproblems; the first is conditional correctness, i.e. correctness under the assumption that the execution of the program terminates, and secondly, the termination of the program. Until further notice this section deals with conditional correctness.

To illustrate the approach we will refer to the simplest language level of section 3.2 , i.e. a fixed set of variables, assignment statement and compound statement. The notation and particulax axioms of the example are due to C.A.R. Hoare. The basic new piece of notation are propositions of the form:

$$
p 1\{s t\} p^{2}
$$

where $p 1$ and $p 2$ are propositions referring to variables of the program, and $s t$ is a statement. The intuitive meaning of the new form is: if $p 1$ is true before the execution of $s t$ and the execution of $s t$ terminates, then $p 2$ is true after the execution of $s t . p 1$ is called precondition, $p 2$ is the so-called postcondition or consequence.

As example for axions and rules of inference we take the assignment 
statement and the compound statement. The axiom (more precisely the axiom schema) for the assignment statement reads:

$$
p_{e}^{x}\{x:=e\} p \text { where } p_{e}^{x} \text { means: replace all occurences of } x \text { in } p \text { by } e \text {. }
$$

In fact, $p_{e}^{x}$ is the weakest possible precondition given $p$ and vice versa given $p_{e}^{x}$ as the precondition $p$ is the strongest possible consequence; i.e. the schema captures all there is to know about the assignment statement. A specific instance of the schema would e.g. be:

$$
0<x+1 \quad\{x:=x+1\} \quad 0<x
$$

Note that in order to use the schema it is not necessary to refer to the denotation of " $x:=x+1$ ". The definition of the compound statement takes the form of a rule of inference and reads:

IF $p 1\left\{s t_{1}\right\} p 2$ AND $p 2\left\{s t_{2}\right\} p 3$

IHEN $p 1\left\{s t_{1} ; s t_{2}\right\} p 3$

For a fuIl language definition there will in general be an axiom per primitive statement and a rule of inference per composite statement; in addition, there are some general rules which have not been exemplified in this section.

The structure of the proofs reflects the syntactic structure of the program text, as one would hope.

There is a simple relation between the discussed axiomatic approach and a corresponding definition á lá mathematical semantics. As already mentioned the axioms and rules of inference can be interpreted as theorems within mathematical semantics. In particular we interpret the new propositional form, $p 1\{s t\} p 2$ as follows. Assume for the moment that pl and $p 2$ are expressions that are also valid expressions in the programming language, denoting truth values.

$$
\begin{aligned}
p 1\{s t\} p 2= & I[p I](\sigma) \supset I[p 2](I[s t](\sigma)) \\
& \text { for all o for which } I[s t] \text { is defined, i.e. } \\
& \text { st terminates. }
\end{aligned}
$$

The various axioms and rules of inference may now be rewritten according to the above interpretation and proven with respect to the definitions of mathematical semantics (see [Manna 72]). 
Neither the generation of the proof nor solving the termination problem can be completely mechanical, since both are in general undecidable. However, there is hope that for frequently occurring program structures the problems can be solved effectively by algorithms. Proposals to solve the termination problem frequently rely on an indirect proof, in particular on finding a quantity which decreases as the computation proceeds, but cannot decrease indefinitely.

The subject of axiomatic definitions and program verification has stimulated widespread research activities due to the intellectually pleasing content but also because of its potential economic value. The belief in the latter is based on the vision that, ultimately, the extremely cost-intensive program testing and so-called program maintenance can be replaced by systematic program design and verification, possibly to some extent automated.

There are many examples of correctness proofs of specific programs (see [Lond on 70]) and several automated verification aids (e.g. [King 75]). The existing examples are mostly small programs for more or less complicated mathematical problems. Some of the algorithms published in the respective section of the CACM are certified by proofs. An attempt to axiomatize a full language, PASCAL, has been undertaken by Hoare and wirth [Hoare 73 ] resulting in the definition of at least a large subset.

Intimately connected to axiom systems for programming languages is the issue of programming style and development methodology. The essence of structured programing is, in the light of the presented research, the recommendation to use only language constructs which have simple axioms (this excludes e.g. the general form of gotos, although restricted forms may well lead to simple correctness arguments). As it turns out, the process of developing a program is intimately connected to the generation of the corresponding correctness proof. Thus we may expect guidance how to develop programs rather than merely learn how to prove ready made programs correct.

Yet, there is an enormous gap between current programming practice and the complexity of the software being produced on the one hand, and the vision and capabilities of the systematic techniques described. The proper discussion of the dilemma needs a larger context than has been given in this section and will therefore be deferred to the next section. 


\section{CHALLENGES}

In accordance with the intent of the entire paper, the scope of this section excludes topics considered to belong to the theory of computation. With this restriction in mind we may certainly say that the definition of programming language semantics is not an end in itself; consequently, the discussion of future directions cannot be isolated from the intended applications of semantic definitions, i.e. precise definition of real life languages, compiler development, program development and language design.

There are two topics we want to clearly separate to avoid a frequent confusion: Firstly, the semantic analysis and formal definition of existing programming languages; secondly, the design of novel, useful language constructs.

Current programming languages are a compromise between the desire to provide the most comfortable and elegant language for the human user and the aim to construct efficient implementations on given systems with known compiler technology. Furthermore, the more intensely used languages undergo an evolution over the years to support new system functions. These languages were not designed with the aim to make formal correctness proofs easy nor were they designed to fit most comfortably into the framework of mathematical semantics. However, it would be a mistake to conclude that these languages are no longer worth the attention of computer science. In view of the heavy investment by users as well as manufacturers it is not likely that the current programing languages will change radically in the near future. Thus the carriers of new programming style will be, at least for some time, current languages. The initial motivation of formal semantics, precise definition to achieve portability, is still valid; there is as yet no semantic analysis of COBOL (the most widely used programing language). A comparative language study on the semantics level would be quite valuable [Strachey 73]. Finally, there is no comprehensive representation of the existing implementation techniques related to formalized semantic concepts.

Whereas BNF, or slight variations thereof, are widely accepted as a means to define a concrete syntax, there is no such widespread consensus for any of the semantic description schemes. 
Next I wish to offer a top down argument to justify the major long range goals of the present subject. Firstly, we can observe that over the past two decades the speed and storage capacity of computers have been increased exponentially roughly at a rate of about 40 percent a year. This trend has been balanced by a similar decrease of cost per operation and per storage unit (i.e. bit or byte). Similarly the size of systemcode (operating system, compilers, etc.) has increased exponentially as well. However, in this case no balancing trend of decreasing cost per line of code can be observed. Furthermore, we will not only have to master greater quantity but larger complexity as well. We conclude that software production is or soon will be the bottleneck for the use of computers.

There are three general research directions promising to improve the situation:

1. Advance Automatic Programming

2. Remove Testing in favor of Correctness Proofs

3. Advance Modular Programming

By the first research area we mean to extrapolate the development of higher level languages by introducing more abstract datatypes (such as e.g. sets) and their associated operations, relax restrictions in current languages and introduce more powerful control structures; the intent is, of course, to automate part of the production process; in short, follow the trends suggested under the term "very high level language". Topics one and two are intimately connected. As J. Schwartz [Schwartz 75] observes, it is much easier to prove the correctness on an abstract level rather than on the level of detalled representations. If the abstract program can be compiled, the task of the programmer is completed, provided the compiler has been proven as well. Thus the step from the abstract algorithm to its ultimate representation in machine form is proven once and for all by a compiler proof. The author believes that research in correctness proof must therefore be investigated hand in hand with the development of very high level languages. Even under the assumption that the level of programming languages can be raised, correctness proofs will remain sufficiently complicated to warrant machine assistance in the form of proof generators and checkers. Although the latter subject has been considerably advanced over the last decade, it has not yet reached the stage of applicability in practical programming. Various subgoals may be envisaged, e.g. conversational systems 
like EFFIGY [King 75] which offer a combination of generalized testing by symbolic execution and some assistance for generating proofs.

A notorious problem in designing large pieces of software is modularity. It is rarely the case that existing modules can be used to build new systems without major trimming, if at all. As J. Dennis [Dennis 75] observes, the success of modular programming not only depends on how modules are written, but also on the characteristics of the linguistic level at which these modules are expressed. This conjecture is supported by a detailed analysis of some high level languages in [Dennis 75 ]. Modules are usually expressed by procedures, subroutines or programs (depending on the specific languages used). In short, we have to look for constructs other than procedures (etc.) and the related traditional ways to compose procedures into larger units, in order to achieve the desired modularity.

After this detour we now return to the subject proper and ask what the relevance of formal semantics with these issues is. Firstly, axiomatic semantics provides the proof theory for program correctness proofs, and thus is also the basis for the mechanical aids in this area. It is rather difficult to propose useful axioms and rules of inference without having an interpreted system first, such as provided by a mathematical or operational system. However, this is a controversial issue.

In search for new language constructs (such as a useful notion of module), formal semantics ought to provide the framework for formulating the problem and for stating and justifying solutions [Strachey 73]. So far most research in formal semantics has been concerned with constructs as found in traditional languages. Here is a piece of language, what does it mean, was the question in the light of the discussed software problems. We should start from the other end, i.e. construct novel denotations and associate a name after we are satisfied with their properties. 